\title{
VAC. 19 - Preliminary Studies of Reactions Using Colominic and Sialic Acids as Prototypes to bivalent vaccine against $S$. agalactiae and $A$. baumannii
}

Marcelio de Moura Oliveira ${ }^{1 \star}$; Marilza B. Correa ${ }^{1}$; Maria de Lourdes M. Leal ${ }^{1}$; Barbara Vasconcellos da Silva ${ }^{1}$; Ellen Jessouroun ${ }^{1}$.

1Fiocruz/Bio-Manguinhos;

2UFRJ - Universidade Federal do Rio de Janeiro.

Introduction: Bioconjugation reactions play an important role in developing molecular arrangement of some different chemical species in new assembled structures. Such structures are of great importance in the vaccinology field against important pathogens. The pathogen S. agalactiae appears as a threat of invasive infection in neonates, characterizing a common problem to several countries. Similarly, the bacterium A. baumannii has been a problem in Brazilian hospitals, mainly due to the increasing development of resistance to different antimicrobial drugs. Therefore, a use of conjugate bivalent vaccines rises as an answer to prevention of nosocomial infections caused by this kind of pathogens. Hence, the development of new chemical approaches for synthesis of bivalent vaccines is imperative. A primary step for development of new methodologies about oxidation using prototypes that mimic functional groups present in S. agalactiae matrix was realized.

Objective: The present work shows the uses of tetrabutylammonium periodate (TBAP), Silicasupported periodate $(\mathrm{SiO} 2 \mathrm{P})$, and sodium periodate $(\mathrm{NaP})$ as oxidant agents with generation of an aldehyde moiety. These reactions have the objective to evaluate regioselectivity and its impact over conjugate reactions, being used as prototypes for specific reactions that will be performed with S. agalactiae polysaccharides as well.

Methodology: TBAP, $\mathrm{SiO} 2 \mathrm{P}$ and $\mathrm{NaP}$ were used to perform $2 \mathrm{MNA}$ oxidation in a proportion of $1: 0.9$ equivalents. TBA and $\mathrm{SiO} 2 \mathrm{P}$ reactions were performed in methanol at room temperature. 2MNA oxidation by $\mathrm{NaP}$ was performed in water and subsequently quenched with sodium sulfide. The TBA and $\mathrm{SiO} 2 \mathrm{P}$ oxidizing products, and $2 \mathrm{MNA}$ reactions, were analyzed by unidimensional NMR techniques. ColA Hydrolysis assay was performed under heterogeneous catalysis/US with action of a strongly acid sulfonic resin (254 mg of $-\mathrm{SO}_{3} \mathrm{H} / 1 \mathrm{~g}$ of resin) in aqueous media. ColA oligosaccharide was analyzed by size exclusion chromatography.

Results: The NMR data showed that oxidation of $2 \mathrm{MNA}$ by SiO2P led to a parcial demethylation (ca. 36\%) of substrate and no oxidation product was observed. TBAP was efficient in oxidizing the 2MNA prototype, evidenced by proton NMR analysis with rising of a sign referent to the aldehyde hidrate at $5.0 \mathrm{ppm}$. ColA hydrolysis showed variations of $\mathrm{K}_{\mathrm{va}}$ values, from size exclusion chromatography. The results of ColA reactions presented its better point at $3 \mathrm{~h}$ of reaction.

Conclusion: The results exhibited a promising methodology for polysaccharide oxidation. The results obtained in the reaction conditions for TBAP indicates that oxidation takes place prevalently between positions $\mathrm{C} 7-\mathrm{C} 8$ of sialic acid moiety. In addition, TBAP is supposed to be an auspicious alternative oxidative methodology besides sodium periodate to use in methanolic media. ColA polysaccharide showed to be a good prototype in exploring reactions using sialic acid as targets.

Keywords: Conjugation; Sialic acid prototype; TBA periodate 